\title{
DSpace@MIT
}

\author{
MIT Open Access Articles
}

\section{Efficient AUV Navigation Fusing Acoustic Ranging and Side-scan Sonar}

The MIT Faculty has made this article openly available. Please share how this access benefits you. Your story matters.

Citation: Fallon, Maurice F. et al. "Efficient AUV Navigation Fusing Acoustic Ranging and Sidescan Sonar" IEEE International Conference on Robotics and Automation. Proceedings, (ICRA), May 9-13, 2011,Shanghai International Conference Center, Shanghai, China.

As Published: https://ras.papercept.net/conferences/scripts/abstract.pl?

ConfID=34\&Number $=1555$

Publisher: IEEE Computer Society

Persistent URL: http://hdl.handle.net/1721.1/64410

Version: Author's final manuscript: final author's manuscript post peer review, without publisher's formatting or copy editing

Terms of use: Creative Commons Attribution-Noncommercial-Share Alike 3.0 


\title{
Efficient AUV Navigation Fusing Acoustic Ranging and Side-scan Sonar
}

\author{
Maurice F. Fallon, Michael Kaess, Hordur Johannsson and John J. Leonard
}

\begin{abstract}
This paper presents an on-line nonlinear least squares algorithm for multi-sensor autonomous underwater vehicle (AUV) navigation. The approach integrates the global constraints of range to and GPS position of a surface vehicle or buoy communicated via acoustic modems and relative pose constraints arising from targets detected in side-scan sonar images. The approach utilizes an efficient optimization algorithm, iSAM, which allows for consistent on-line estimation of the entire set of trajectory constraints. The optimized trajectory can then be used to more accurately navigate the AUV, to extend mission duration, and to avoid GPS surfacing. As iSAM provides efficient access to the marginal covariances of previously observed features, automatic data association is greatly simplified - particularly in sparse marine environments. A key feature of our approach is its intended scalability to single surface sensor (a vehicle or buoy) broadcasting its GPS position and simultaneous one-way travel time range (OWTT) to multiple AUVs. We discuss why our approach is scalable as well as robust to modem transmission failure. Results are provided for an ocean experiment using a Hydroid REMUS 100 AUV co-operating with one of two craft: an autonomous surface vehicle (ASV) and a manned support vessel. During these experiments the ranging portion of the algorithm ran online on-board the AUV. Extension of the paradigm to multiple missions via the optimization of successive survey missions (and the resultant sonar mosaics) is also demonstrated.
\end{abstract}

\section{INTRODUCTION}

Modern Autonomous Underwater Vehicles (AUVs) are complex robotic systems containing several proprioceptive sensors such as compasses, fiber optic gyroscopes (FOG) and Doppler Velocity Loggers (DVL) [1]. The resultant sensor output can be combined together using navigation filters, such as the Extended Kalman Filter (EKF), to produce a high quality estimate of the AUV position and uncertainty. This estimate is then used by the AUV to inform on-board decision making logic and to adaptively complete complex survey and security missions. A recent survey by Kinsey et al. provides a good overview of the state of the art [2].

In addition, many AUVs have installed multiple exteroceptive sensors. Side-scan sonar, initially developed by the US Navy, has been widely used for ship, ROV and AUV survey since its invention in the 1950s. More recently, forward looking sonars, with the ability to accurately position a field of features in two dimensions, have also been deployed for a variety of applications such as 3-D reconstruction [3], ship hull inspection [4] and harbor security [5]. In scenarios in which water turbidity is not excessively high, cameras have been used to produce accurate maps of ship-wrecks and

The authors are with the Computer Science and Artificial Intelligence Laboratory, Massachusetts Institute of Technology, Cambridge, MA, USA mfallon, kaess, hordurj, jleonard@mit.edu

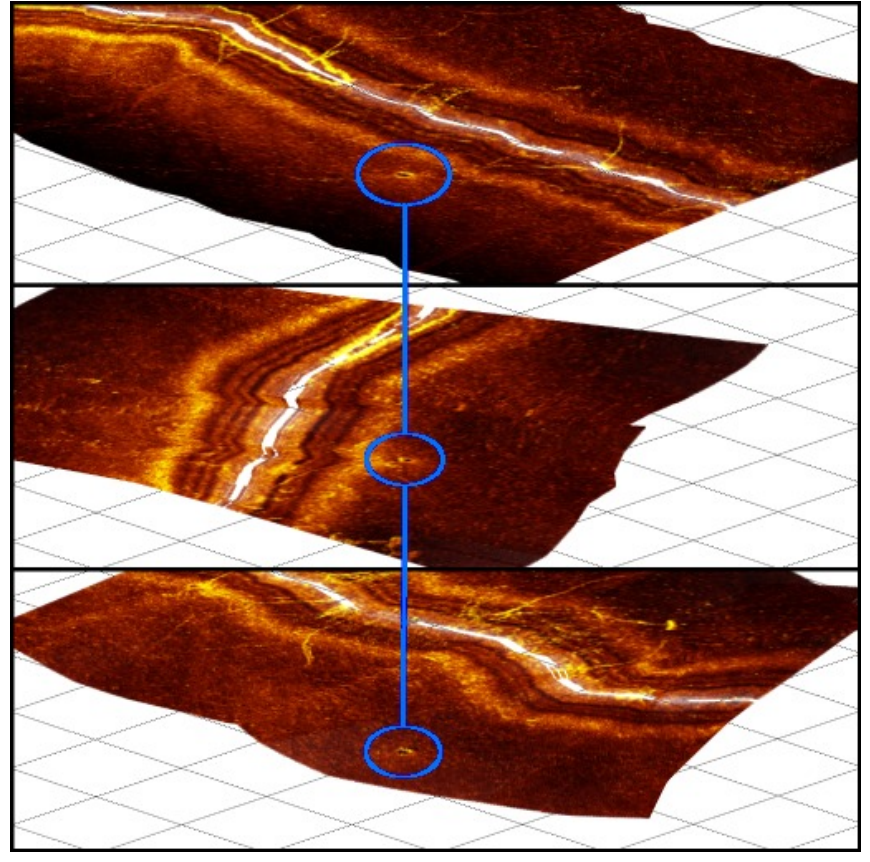

Fig. 1. Optimizing the entire vehicle trajectory and target observation set facilitates explicit alignment of sonar mosaics. In this figure this alignment has been carried out for three different observations of a single target corresponding to Mission 3 mentioned in Sec. 6(a).

underwater historical structures, for example the mapping of RMS Titanic [6] and of Iron Age shipwrecks [7].

Typically the former approaches are intended to estimate the (current) absolute georeferenced vehicle position while the latter approaches can create locally consistent maps using relative measurements. Traditional acoustic ranging, using Long Baseline (LBL) and Ultra Short Baseline (USBL), is commonly used to combine these two modes [8], [9], but they suffer from some operational issues (more fully discussed in Section II-A). Meanwhile advances in one-way travel time ranging (OWTT) using acoustic data modems, such as with the WHOI Micromodem [10], allows for a more flexible type of multi-vehicle navigation.

This paper extends upon previous OWTT navigation research as follows. Initially, the Moving Long Baseline (MLBL) concept used two mobile Autonomous Surface Vehicles (ASVs) to estimate the position of the AUV using acoustic modem ranging. This was proposed by Vaganay et al. [11] and later developed by Bahr [12]. More recently research has focused on utilizing only a single surface vehicle to support a single AUV using a recursive state estimator such as the Extended Kalman filter ([13]) or the Distributed 
Extended Information Filter (DEIF, [14]).

For many robotic applications, however, estimating the vehicle's entire trajectory as well as the location of any observed features is important (for example in feature-based AUV navigation) and is known as Simultaneous Localization and Mapping (SLAM). The EKF has been shown to provide an inconsistent SLAM solution due to information lost during the linearization step [15]. Furthermore, our previous work, [16], demonstrated (off-line) the superior performance of NLS methods in the acoustic ranging problem domain versus both an EKF and a particle filtering implementation although requiring growing computational resources.

Recently developed Nonlinear Least Squares solutions to SLAM have overcome these issues by recovering an exact solution while avoiding the repeated solution of subproblems - providing for the rapid and efficient solution of very large data-sets. Incremental Smoothing and Mapping (iSAM) is representative of the current state-of-the-art in pose graph estimation for SLAM, and it is the core estimation algorithm used in this work.

In this paper, we utilize iSAM both for pose estimation using acoustic range data, and also concurrently for mapping and localization of bottom targets identified in side-scan sonar imagery. As an extension, we demonstrate the ability to combine relative constraints across successive missions, enabling multi-session AUV navigation and mapping, in which data collected in previous missions is seamlessly integrated on-line with data from the current mission onboard the AUV.

Considering previous related research in AUV navigation and mapping, the work of Tena Ruiz et al. [17], [18] is highly relevant. This work demonstrated SLAM using side-scan sonar, comparing the performance of a standard EKF with use of the the Rauch-Tung-Streibel Kalman smoother. Their results demonstrate the superiority of a smoothing approach when applied to AUV side-scan mapping. Our work advances on this work by applying a state-of-the-art pose graph SLAM state estimation and integrating acoustic range measurements from a surface vehicle, which has not to our knowledge been addressed in previous research.

Other related research that has considered acoustic range data to stationary beacons in a smoothing framework include [19], [20]. Finally, vision-based AUV navigation has employed many techniques similar to those discussed here. In many ways this modality can be complementary to sonar navigation for example providing higher fidelity with reduced sensor range, for example [21], [22].

Section II gives an overview of the sensor geometry before presenting the vehicle and sensor measurement models within a common framework. This section also discusses design issues for an acoustic ranging system which can support any number of AUVs listening to the broadcasted surface positions. Section III gives an overview of the adaptation of the iSAM estimation algorithm to this problem while also discussing the considerations for multi-vehicle mapping. Presented in Section IV is a demonstration of how a rich multi-sensor, multi-vehicle four mission dataset could be combined into a single navigation problem which can be solved in realtime on-board the AUV.

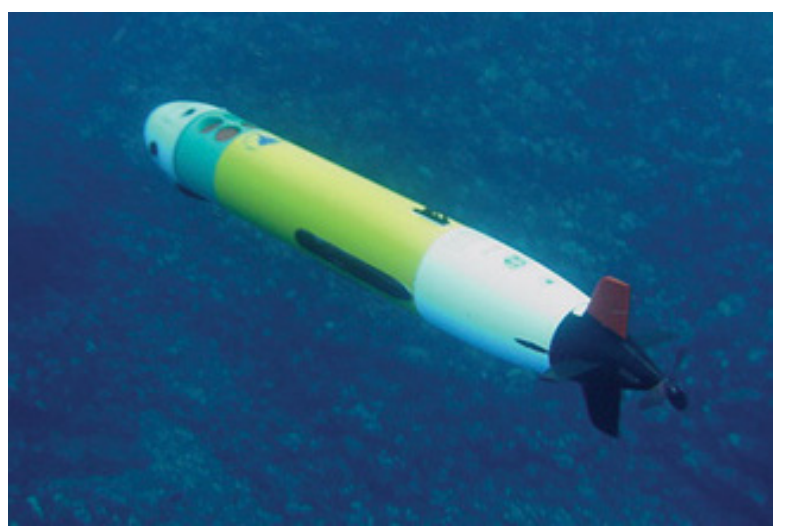

(a)
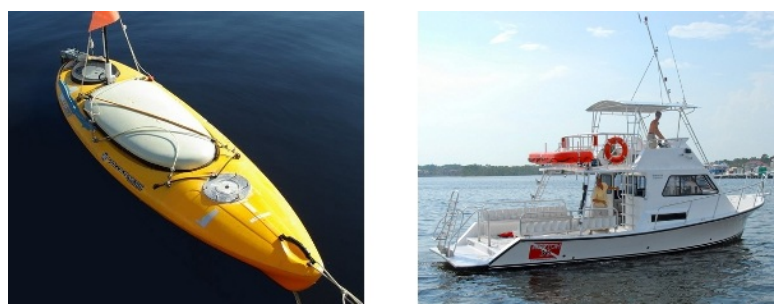

(b)

Fig. 2. The vehicles used in our experiments: the Hydroid Remus 100 AUV was supported by the MIT Scout ASV or our research vessel the Steel Slinger.

\section{Measurement Model}

The full vehicle state is defined in three Cartesian and three rotation dimensions, $[x, y, z, \phi, \theta, \psi]$. Absolute measurements of the depth $z$, roll $\phi$ and pitch $\theta$, are measured using a water pressure sensor and inertial sensors. This leaves three dimensions of the vehicle to be estimated in the horizontal plane: $x, y, \psi$.

The heading is instrumented directly using a compass and this information is integrated with inertial velocity measurements to propagate estimates of the $x$ and $y$ position ${ }^{1}$. This integration is carried out at a high frequency $(\sim 10 \mathrm{~Hz})$ compared to the exteroceptive range and sonar measurements, $\mathcal{O}(1 \mathrm{~min})$.

Following the formulation in [23], [24], the motion of the vehicle is described by a Gaussian process model as follows

$$
x_{i}=f\left(x_{i-1}, u_{i}\right)+w_{i} \quad w_{i} \sim N\left(0, \Sigma_{i}\right)
$$

where $x_{i}$ represents the 3-D vehicle state (as distinct from the dimension $x$ above).

\section{A. Acoustic Ranging}

Acoustic Ranging has been widely used to contribute to AUV navigation [25], [26]. LBL navigation was initially developed in the 1970's [27], [28] and is commonly used

\footnotetext{
${ }^{1}$ In our case this integration is carried out on a separate proprietary vehicle control computer and the result is passed to the payload computer.
} 
by industrial practitioners. It requires the installation of stationary beacons at known locations surrounding the area of interest which measure round-trip acoustic time of flight before triangulating for 3D position estimation. Operating areas are typically restricted to a few $\mathrm{km}^{2}$.

USBL navigation is an alternative method which is typically used for tracking an underwater vehicle's position from a surface ship. Range is measured via time of flight to a single beacon while bearing is estimated using an array of multiple hydrophones on the surface vehicle transducer. Overall position accuracy is highly dependent on many factors, including the range of the vehicle from the surface ship, the motion of the surface ship, and acoustic propagation conditions.

Instead of either LBL or USBL, our work aims to utilize acoustic modems, such as the WHOI Micro-Modem [29], which are already installed on the majority of AUVs for command and control. The most accurate inter-vehicle ranging is through one-way travel time ranging with precisely synchronized clocks, for example using the design by Eustice [30], which also allows for broadcast ranging to any number of vehicles in the vicinity of the transmitting vehicle. An alternative is round trip ranging (RTR), which while resulting in more complexity during operation and higher variance, requires no modification of existing vehicles.

Regardless of the ranging method, the range measurement $r_{j, 3 D}$, the 2-D position of the transmitting beacon, $b_{j}=$ $\left[x_{b j}, y_{b j}\right]$, and associated covariances will be known to the AUV at intervals on the order of 10-120 seconds. Having transformed the range to a 2-D range over ground $r_{j}$ (using the directly instrumented depth), a measurement model can be defined as follows

$$
r_{j}=d\left(x_{j}, b_{j}\right)+\mu_{j} \quad \mu_{j} \sim N\left(0, \Xi_{j}\right)
$$

where $x_{j}$ represents the position of AUV state at that time. GPS measurements of the beacon position are assumed to be distributed via a normal distribution represented by $\Phi_{j}$.

Comparing the on-board position estimates of the AUV and the ASV in the experiments in Section IV, round trip ranging is estimated to have a variance of approximately 7 meters, compared with a variance of 3 meters for oneway ranging reported in [16]. An extra issue is that with the ranging measurement occurring as much as 10 seconds before the position and range are transmitted to the AUV, an acausal update of the vehicle position estimate is required.

The operational framework used by Webster et al. [14], [31] is quite similar to ours. Their approach is based on a decentralized estimation algorithm that jointly estimates the AUV and a supporting research vessel positions using a distributed extended information filter. Incremental updates of the surface vehicle's position are integrated into the AUVbased portion of the filter via a simple and compact addition which, it is assumed, can be packaged within a single modem data packet.

This precise approach hypothesizes the use of a surface vehicle equipped with a high accuracy gyrocompass and a survey-grade GPS (order of $50 \mathrm{~cm}$ accuracy). Furthermore, as described in [31], the approach can be vulnerable to packet loss, resulting in missing incremental updates which would cause the navigation algorithm to fail. While re-broadcasting strategies to correct for such a failure could be envisaged, it is likely that significant (scarce) bandwidth would be sacrificed, making multi-vehicle operations difficult.

Our approach instead aims to provide independent surface measurements to the AUV in a manner that is robust to inevitable acoustic modem packet loss. The goal is a flexible and scalable approach that fully exploits the one-way travel time ranging data that the acoustic modems enable. The solution should be applicable to situations in which only low-cost GPS sensors are available on the ASVs or gateway buoys, to provide maximum flexibility.

\section{B. Side-scan Sonar}

Side-scan sonar is a common sonar sensor often used for ocean seafloor mapping [32]. As the name suggests, the sonar transducer device scans laterally when towed behind a ship or flown attached to an AUV through the water column. A series of acoustic pings are transmitted and the amplitude and timing of the returns combined with speed of sound in water is used to determine the existence of features located perpendicular to the direction of motion.

By the motion of the transducer through the water column, two-dimensional images can be produced which survey the ocean floor and features on it. See Fig. 3 for an example sidescan sonar image. These images, while seemingly indicative of what exists on the ocean floor, contain no localization information to register them with either a relative or global position. Also it is often difficult to repeatedly detect and recognize features of interest, for example, Fig. 3 illustrates two observations each of two different targets of interest. Target 1 (a metallic icosahedron) appears differently in its two observations. Targets are typically not identified using the returned echoes from the target itself, but by the shadow cast by the target [32].

For these reasons we must be careful in choosing side-scan sonar features for loop closure. Appearance-based matching techniques, such as FABMAP [33], would most likely encounter difficulties with acoustic imagery. Metric-based feature matching requires access to accurate, fully optimized position and uncertainty estimates of the new target relative to all previously observed candidate features. For these reasons, we propose to use an efficient on-line smoothing and mapping algorithm, iSAM [23], to optimize the position and uncertainty of the entire vehicle trajectory, the sonar target positions, as well as all the beacon range estimates mentioned in Section II-A.

The geometry of the side-scan sonar target positioning is illustrated in Fig. 3. Distance from the side-scan sonar to a feature corresponds to the slant range, $d_{m, 3 \mathrm{D}}$, while the distance of the AUV off the ocean floor (altitude, $a_{m}$ ) can be instrumented. We will assume the ocean floor to be locally flat $^{2}$ which allows the slant range to be converted into the

\footnotetext{
${ }^{2}$ In the experiments presented in Section IV, the ocean floor had a gradient of $0.5 \%$ - justifying this assumption.
} 
horizontal range, resulting in the following relative position measurement

$$
\begin{aligned}
d_{m, 2 \mathrm{D}} & =\sqrt{d_{m, 3 \mathrm{D}}^{2}-a_{m}^{2}} \\
\psi_{m} & = \pm \pi / 2
\end{aligned}
$$

where $\psi_{m}$ is the bearing to the target defined from the front of the vehicle anti-clockwise. These two measurements paired together give a relative position constraint, $z_{m}=$ $\left[d_{m, 2 \mathrm{D}}, \psi_{m}\right]$ for an observation of target $s_{m}$. This target can either be a new, previously unseen target or a re-observation of an older target. In the experiments in Section IV this data association is done manually while in future work we will aim to do this automatically as in [18]. The resultant measurement model will be as follows

$$
z_{m}=h\left(x_{m}, s_{m}\right)+v_{m} \quad v_{m} \sim N\left(0, \Lambda_{m}\right)
$$

where $x_{m}$ is the pose of the AUV at that time. In effect, repeated observations of the same sonar target correspond to loop-closures. Such repeated observations of the same location allow uncertainty to be bounded for the navigation between the observations.

\section{SMOOTHING AND MAPPING}

The overall measurement system is described by the factor graph in Fig. 4. The joint probability distribution of the vehicle trajectory, $X=\left[x_{1} x_{2} \ldots x_{N}\right]$; acoustic range measurements, $R=\left[\begin{array}{llll}r_{1} & r_{2} & \ldots & r_{J}\end{array}\right]$; relative sonar measurements, $Z=\left[\begin{array}{llll}z_{1} & z_{2} & \ldots & z_{M}\end{array}\right]$; and the set of control measurements between two successive poses $U=\left[\begin{array}{lllll}u_{1} & u_{2} & \ldots & u_{N}\end{array}\right]$ is given by

$$
\begin{array}{r}
P(R, Z, U, X)=P\left(x_{0}\right) \prod_{i=1}^{N} P\left(x_{i} \mid x_{i-1}, u_{i}\right) \\
\prod_{j=1}^{J} P\left(g_{j}\right) \prod_{j=1}^{J} P\left(r_{j} \mid x_{j}, b_{j}\right) \prod_{m=1}^{M} P\left(z_{m} \mid x_{m}, s_{m}\right)
\end{array}
$$

where $x_{j}$ represents the vehicle pose when measuring the range $r_{j}$ to beacon $b_{j}$, and $x_{m}$ when observing sonar target $s_{m}$ at relative position $z_{m}$. This maintains the approach presented in [23], [24]. Note that each beacon position $b_{j}$ is initialized using its GPS measurment $g_{j}$ as a prior $P\left(g_{j}\right)$, as indicated in Figure 4, which implicitly assumes successive measurements to be independent which is important to maintain flexibility.

Our specific implementation incorporated absolute measurements from a proprietary INS system in the global frame. For this reason we also required a prior on the vehicle heading $\psi_{i}$ which has been omitted here for clarity.

A maximum a posteriori (MAP) estimate of the vehicle trajectory, $\mathrm{X}$, can be formed given the measurements $\mathrm{R}$, $\mathrm{Z}$, and $\mathrm{U}$. Denoting this estimate $\hat{X}$, the resultant MAP

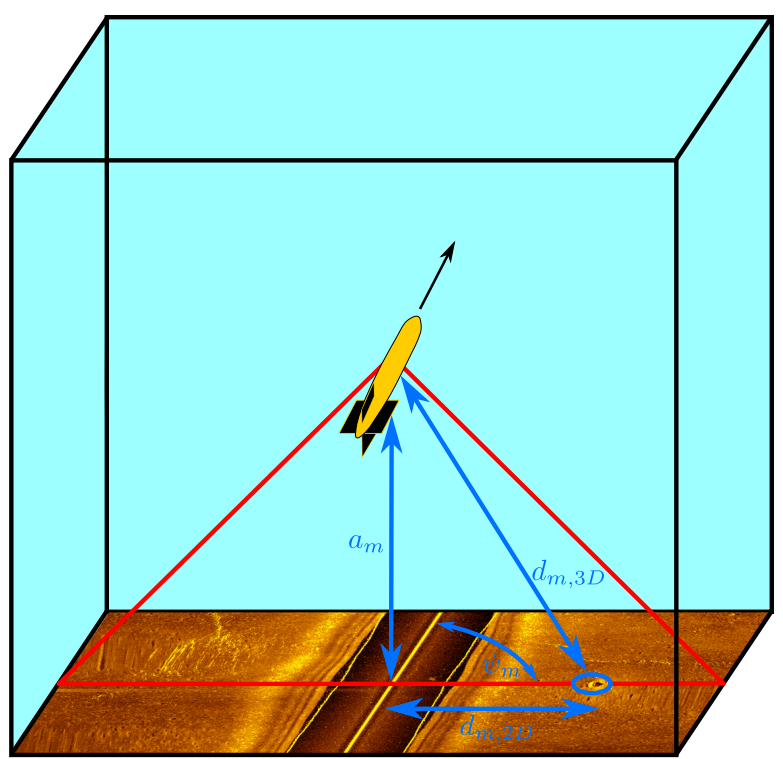

(a)

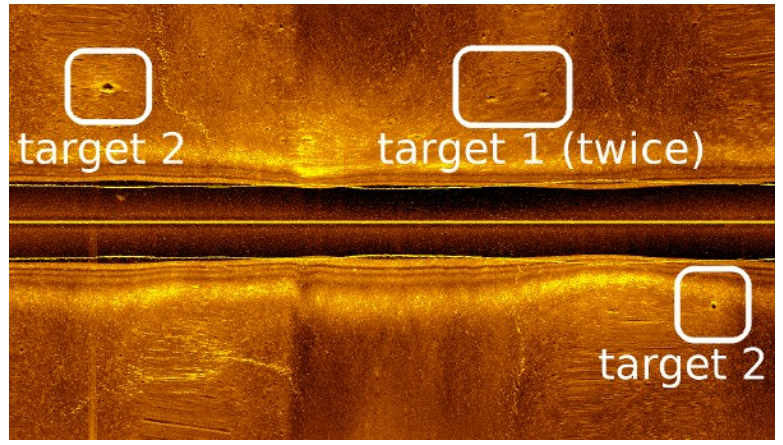

(b)

Fig. 3. (a): As the AUV travels through the water the side-scan sonar images laterally with objects on the ocean floor giving strong returns. (b): A top down projection of the side-scan sonar for a $120 \mathrm{~m}$ of vehicle motion (left to right). The lateral scale is $30 \mathrm{~m}$ in each direction which yields a 1:1 aspect ratio. Note that in this case Targets 1 and 2 have been observed twice each after an about turn.

estimator is given by

$$
\begin{aligned}
\hat{X} & =\underset{X}{\arg \max } P(R, Z, U \mid X) P(X) \\
& =\underset{X}{\arg \max } P(R, Z, U, X) \\
& =\underset{X}{\arg \min }-\log P(R, Z, U, X)
\end{aligned}
$$

Assuming Gaussian measurement noise and using the process and measurement models defined in preceding sections, we arrive at the following nonlinear least-squares problem

$$
\begin{aligned}
\hat{X}= & \underset{X}{\arg \min } \sum_{i=1}^{N}\left\|f\left(x_{i-1}, u_{i}\right)-x_{i}\right\|_{\Sigma_{i}}^{2} \\
& +\sum_{j=1}^{J}\left\|b_{j}-\hat{g}_{j}\right\|_{\Phi_{j}}^{2}+\sum_{j=1}^{J}\left\|d\left(x_{j}, b_{j}\right)-\hat{r}_{j}\right\|_{\Xi_{j}}^{2} \\
& +\sum_{m=1}^{M}\left\|h\left(x_{m}, s_{m}\right)-\hat{z}_{m}\right\|_{\Lambda_{m}}^{2}
\end{aligned}
$$




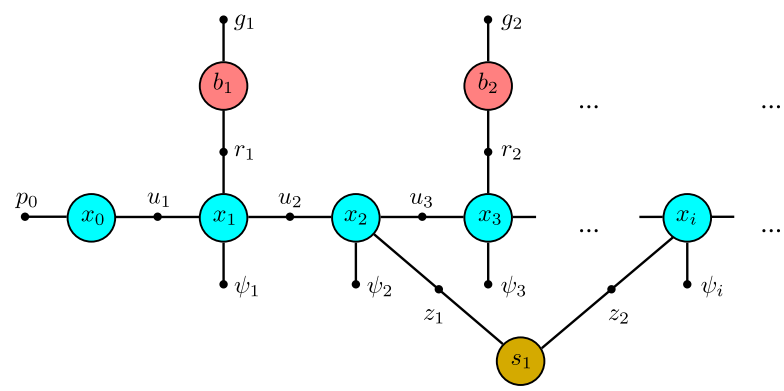

Fig. 4. Factor graph formulation of the measurement system showing vehicle states $x_{i}$, surface beacons $b_{j}$ and sonar targets $s_{k}$. Also illustrated are the respective constraints: range $r_{j}$ in the case of the surface beacons and range and relative bearing $z_{m}$ in the case of sonar targets. Ranges are paired with surface beacon measurements while multiple observations of a particular sonar target is in effect a loop closure

where $\|x\|_{\Sigma}^{2}:=x^{T} \Sigma x$.

The resultant problem is sparse, which lends itself to solution using incremental Gauss-Newton methods such as Incremental Smoothing and Mapping (iSAM) [23]. This approach updates the entire vehicle trajectory and landmark set when new measurements are received rather than recalculating the nonlinear least squares system anew each iteration. Computation analysis presented therein indicate that this approach could run in real-time for missions of our type for tens hours, assuming that range and/or sonar measurements are obtained approximately once per minute.

\section{A. Multi-Mission Operation}

While SLAM algorithms are most commonly utilized on a single vehicle during operation, extensions have been developed to support operation across multiple robots or missions [34], [35] for faster or persistent operation. As GPS is typically not assumed, careful consideration of robot-torobot encounters so as to combine the vehicle maps is a key consideration. In the AUV domain, however, operation is explicitly within the global coordinate frame due to initialization at known GPS positions (with some uncertainty) and the use of a compass or a fiber optic gyroscope (FOG) for heading measurement. However, simultaneous multiple AUV navigation has only begun to be investigated due to low acoustic communication bandwidth [36] as well as the high cost of operating the vehicles.

Instead, we consider the optimization of successive missions with a single AUV in the same part of the ocean. This is important because many AUV applications require either revisiting previously surveyed locations to more closely observe targets of interest, or resurveying to detect changing environments over time.

The problem will be proposed as the joint optimization of two vehicle trajectories which are independent - except for the observation of the same sonar target across different missions. This requires only minor indexing modification of Eq. 10. The individual portions of the resulting combined map will in fact be more accurate than individual optimization alone. Computationally, the optimization will be no more intensive than a single extended mission. (See [34] for a related discussion.) This approach is demonstrated in Section 6(a).

\section{EXPERIMENTS}

A series of experiments were carried out in St. Andrews Bay in Panama City, Florida to demonstrate the proposed approach. A Hydroid REMUS 100 AUV carried out four different missions while collecting side-scan sonar data (using a Marine Sonics transducer) as well as range and GPS position information transmitted from either the Scout ASV (Fig.2) or a deck-box on the $10 \mathrm{~m}$ support vessel. In each case a low cost Garmin 18x GPS Sensor was used to provide GPS position estimates.

The Kearfott T16 INS, connected to the REMUS frontseat computer, fused its RLG measurements with those of a Teledyne RDI DVL, an accelometer and a GPS sensor to produce excellent navigation performance. For example after a 40 minute mission the AUV surfaced with a $2 \mathrm{~m}$ GPS correction - drift of the order of $0.1 \%$ of the distance traveled.

The AUV did not have the ability to carry out one-way ranging and as a result two-way ranging was used instead. The navigation estimate was made available to a backseat computer which ran an implementation of the algorithm in Sec. III (less the sonar portion).

Given the variance of two-way ranging $(\sim 7 \mathrm{~m})$ and the accuracy of the vehicle INS, it would be ambitious to expect to demonstrate significant improvement using cooperative ranging-assisted navigation in this case. For this reason these missions primarily present an opportunity to validate and demonstrate the system with combined sensor input and multiple mission operation. As stated previously, the intended application area of this technology is not in the improvement of the performance of short (hour-long) missions with such an AUV but rather for very long duration missions $(\sim 10 \mathrm{hrs})$ or with much less accurate AUVs.

Given these issues, we estimate that the resultant bounded error for a non-RLG enabled AUV with several percent drift would be of the order of 3-5m (depending on the relative geometry and frequency of the OWTT range measurements). Future work will aim to properly quantify this value on such a platform.

For simplicity we will primarily focus on the longest mission - Mission 3 in Fig. 6(a) - before discussing the extension to successive missions in Section IV-B. The missions are numbered chronologically.

\section{A. Single Mission}

During Mission 3, the AUV navigation data was combined with the acoustic range/position pairs and optimized online on-board the AUV using iSAM to produce a realtime estimate of its position and uncertainty. After the experiments, sonar targets were manually extracted from the Marine Sonics data file and used in combination with the other navigation data to produce the combined optimization illustrated in Fig. 5. An overview of the mission is presented 
in Fig. 6 as well as quantitative results from the optimization where $3 \sigma$ uncertainty was determined using $3 \sqrt{\sigma_{x}^{2}+\sigma_{y}^{2}}$.

Starting at $(400,250)$, the vehicle carried out a set of four re-identification (RID) patterns. These overlapping patterns are designed to provide multiple opportunities to observe objects on the ocean floor using the side-scan sonar. Typically this mission is carried out after having first coarsely surveyed the entire ocean floor. In this case two artificial targets were placed at the center of patterns 2 and 3 and were detected between 15-24 mins (6 times) and 27-36 mins (7 times) respectively. The surface beacon, in this case the support vessel on anchor at $(400,250)$, transmitted round-trip ranges to the AUV on a 20 second cycle.

A quantitative analysis of the approach is presented in Fig. 6(a). The typical case (black) of using only dead reckoning for navigation results in ever increasing uncertainty. The second approach (blue) utilizes target re-identifications in the sonar data but not acoustic range measurements. This temporarily halts the growth of uncertainty but monotonic growth continues in their absence.

Acoustic ranging by comparison (red) can achieve bounded error navigation - in this case with a $3 \sigma$-bound of about $2 \mathrm{~m}$. As the AUV's mission encircled the support vessel, sufficient observability was achieved to properly estimate the AUV's state - which results in the changing alignment of the uncertainty function. However performance deteriorates when the relative positions of the vehicles do not vary significantly (such as during patterns 3-4; 40-53 mins).

Finally, the best performance is observed when the sonar and acoustic ranging data are fully fused. Interestingly, the two modalities complement each other: during reidentification patterns 2 and 3, sonar target observations bound the uncertainty while the AUV does not move relative to the support vessel. Later the vehicle transits between patterns - allowing for the range observability to improve.

Note that the initial fall in uncertainty is due to the algorithm being initialized with a conservative initial covariance of $5 \mathrm{~m}$ in each direction. Also the covariances presented in Fig. 6(a) are for the fully converged system at the end of the mission. A full-trajectory optimization of the AUV pose would have been available to the AUV for path planning during operation via iSAM, this estimate would later have been improved upon using as yet unreceived measurements to generate Fig. 6(a). See [16] for more information.

In summary, the combination of the on-board, sonar and ranging sensor measurements allows for on-line navigation to be both globally bounded and locally drift-free.

\section{B. Multiple Missions}

In this section we will describe how the algorithm has been extended to combine the maps produced by multiple successive AUV missions within a single optimization framework. As mentioned in previous sections, it is advantageous to provide a robot with as much prior information of its environment before it begins its mission, which it can then improve on as it navigates.
Space considerations do not permit a full analysis of this feature, but briefly: during Missions 1 and 2 surface information was transmitted from an Autonomous Surface Vehicle, MIT's Scout kayak (shown in Fig. 2), which moved around the AUV so as to improve the observability of the AUV, as previously demonstrated in [16]. In Mission 4, as in Mission 3, the support vessel was instead used - although in this case the support vessel moved from a location due east of the AUV to another location due west of the AUV, as illustrated in Fig. 5. This demonstrates that a basic maneuver by the support vessel is sufficient to ensure mission observability. The mission started at $(350,200)$.

While no quantitative results of the 4-mission optimization are presented here, Fig. 6(b) illustrates the inter-mission connectivity. This demonstrates that the two targets were observed numerous times during the missions, which allows us to combine the navigation across all of the missions into a single fully optimized estimate of the entire operation area. Note that targets seen on only one or two occasions are not presented for clarity.

While such an approach could possibly be carried out for several vehicles operating simultaneously, sharing minimal versions of their respective maps [36], it is unclear if the acoustic bandwidth available would be sufficient to share sonar target observation thumbnails to verify loop closure.

\section{CONCLUSIONS AND FUTURE WORK}

The paper has presented a method for the fusion of onboard proprioceptive navigation and relative sonar observations with acoustic ranges transmitted from an autonomous surface vehicle. The approach is optimal and consistent while maintaining computational efficiency, which allows for operation for many hours in real-time for missions of the type described above.

Factors resulting in a reduction in performance of this approach are as follows: (1) infrequent ranging (2) ranging from the same relative direction (3) sonar targets not being present or being infrequently observed.

Future work will focus on the implementation of this approach combined with on-board decision making which will allow the AUV to maintain accurate navigation so called active localization. While the ranging portion of the algorithm operated entirely online on-board an AUV, accurate on-line detection of sidescan sonar targets will also be required using an approach similar to [18], [24].

This approach will again utilize a Hydroid REMUS 100, but specifically the vehicle will not have a laser gyroscope resulting in on-board navigation which will be less accurate but of a much more typical performance. One-way ranging will also be supported - giving more balanced sensor inputs and allowing each modalities to contribute evenly.

Multi-AUV cooperative navigation (without a surface node) is substantially different to the problem discussed here, the solution of which requires careful consideration of intervehicle correlation in the context of extremely limited underwater bandwidth. 


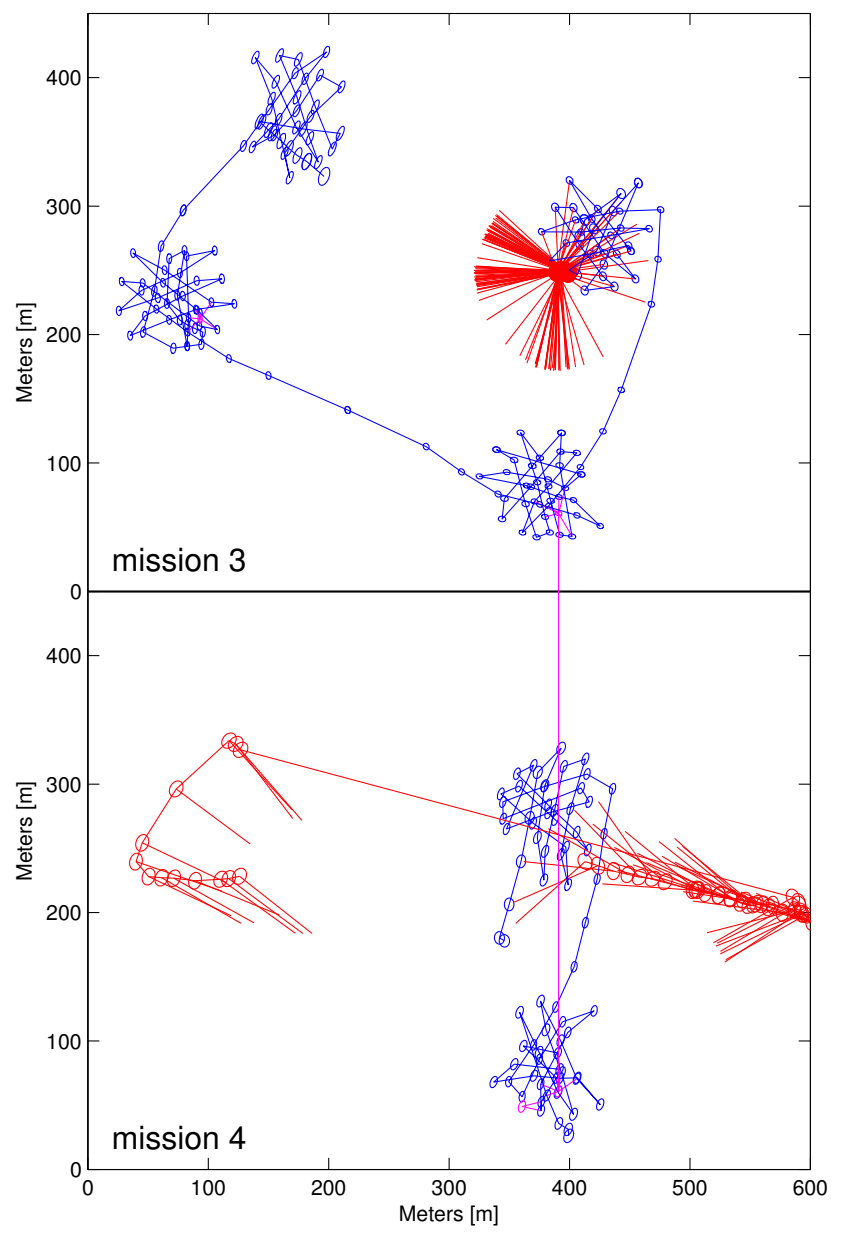

Fig. 5. An overview of the optimized trajectory estimates of the AUV (blue) and the surface vehicle (red), as well as the estimated position of three sonar targets (magenta) for two of the missions. Note that the single magenta line between the two figures demonstrates the mutually observed target which allows for the joint optimization of the two missions. This corresponds to Target 3 in Figure 6(b). The red lines indicate the relative vehicle positions during ranging while the ellipses indicate position uncertainty.

\section{ACKNOWLEDGMENTS}

This work was supported by ONR Grant N000140711102. The authors wish to thank Georgios Papadopoulos for initial contributions to the NLS work and Michael Benjamin, Joseph Curcio, Taylor Gilbert, Andrew Bouchard (NSWCPC) and Jason Price (NSWC-PC) for their help with the collection of the experimental data.

This work utilizes Incremental Smoothing and Mapping (iSAM): an optimization library for sparse nonlinear problems - such as SLAM. iSAM is available to download from:

http://people.csail.mit.edu/kaess/isam/

It also utilizes MOOS-IvP [37] for inter-process communication and autonomous decision making and the gobyacomms acoustic networking libraries [38].

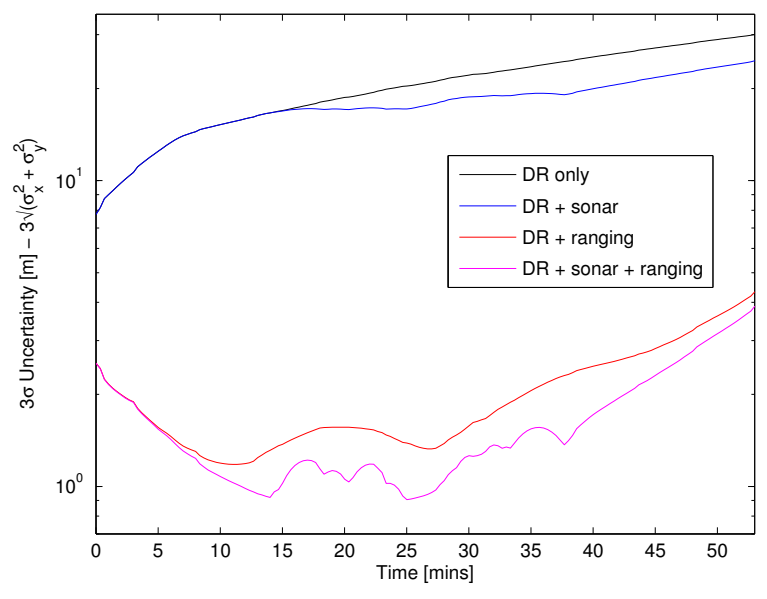

(a)

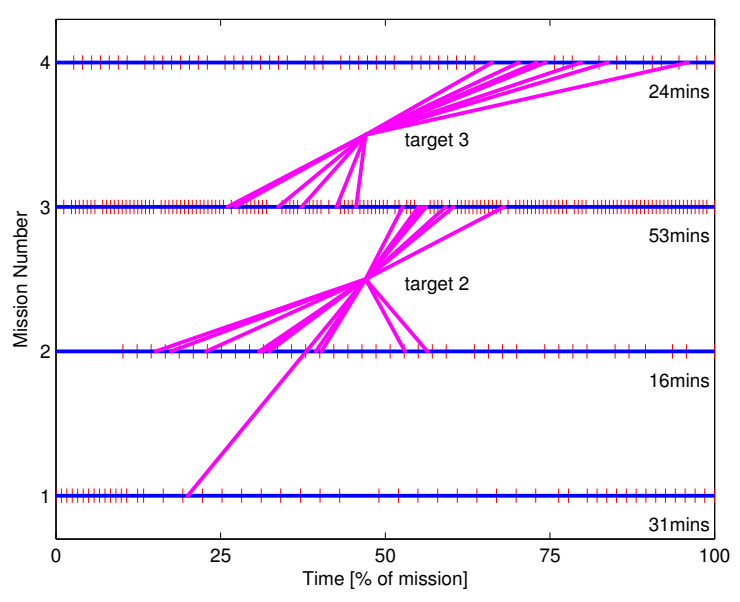

(b)

Fig. 6. (a): Navigation uncertainty for Mission 3 for four different algorithm configurations. As expected the dead reckoning-only solution suffers from continuous uncertainty growth (black). When sonar targets are observed, uncertainty growth can be halted using loop closures, before returning to dead reckoning (blue). Acoustic ranging alone can bound error growth - subject to observability (red); while the full sensor fusion produces the solution with minimum uncertainty (magenta). See Sec. 6(a) for more details. (b): During the four (consecutive) missions, range measurements (represented by the red lines) were frequently received from the ASV (Mission 1 and 2) or the research vessel (Mission 3 and 4). Occasionally targets were detected in the side-scan sonar data. Repeated observations of the same target (illustrated in magenta) allow for a SLAM loop closure and for inter-loop uncertainty to be bounded. Note that multiple observations of the same targets occur across the four missions enabling multi-session mapping. Finally, the events presented for Mission 3 synchronize with Fig. (a).

\section{REFERENCES}

[1] L. Whitcomb, D. Yoerger, and H. Singh, "Advances in dopplerbased navigation of underwater robotic vehicles," in Robotics and Automation, 1999. Proceedings. 1999 IEEE International Conference on, vol. 1, pp. $399-406$ vol.1, 1999.

[2] J. C. Kinsey, R. M. Eustice, and L. L. Whitcomb, "A survey of underwater vehicle navigation: Recent advances and new challenges," in IFAC Conference of Manoeuvering and Control of Marine Craft, (Lisbon, Portugal), September 2006. Invited paper.

[3] D. P. Horner, N. McChesney, T. Masek, and S. P. Kragelund, "3D 
reconstruction with an AUV mounted forward looking sonar," in Proc. Int. Symp. on Unmanned Untethered Submersible Technology, pp. 1464-1470, Aug. 2009.

[4] B. Englot, H. Johannsson, and F. Hover, "Perception, stability analysis, and motion planning for autonomous ship hull inspection," in Proceedings of the International Symposium on Unmanned Untethered Submersible Technology (UUST), 2009.

[5] D. Ribas, P. Ridao, J. Neira, and J. Tardós, "SLAM using an imaging sonar for partially structured underwater environments," in IEEE/RSJ Intl. Conf. on Intelligent Robots and Systems (IROS), 2006.

[6] R. Eustice, H. Singh, J. Leonard, M. Walter, and R. Ballard, "Visually navigating the RMS Titanic with SLAM information filters," in Robotics: Science and Systems (RSS), Jun 2005.

[7] R. D. Ballard, L. E. Stager, D. Master, D. Yoerger, D. Mindell, L. L. Whitcomb, H. Singh, and D. Piechota, "Iron Age shipwrecks in deep water off Ashkelon, Israel," American Journal of Archaeology, vol. 106, pp. 151-168, March 2002.

[8] B. J. M. Mandt, K. Gade, "Integrating DGPS-USBL positioning measurements with inertial navigation in the HUGIN 3000 AUV," in Saint Petersburg International Conference on Integrated Navigation Systems, (Russia), May 2001.

[9] P. Rigby, O. Pizarro, and S. B. Williams, "Towards geo-referenced AUV navigation through fusion of USBL and DVL measurements," in Proceedings of the IEEE/MTS OCEANS Conference and Exhibition, Sep 2006.

[10] R. Eustice, L. Whitcomb, H. Singh, and M. Grund, "Recent advances in synchronous-clock one-way-travel-time acoustic navigation," in Proceedings of the IEEE/MTS OCEANS Conference and Exhibition, (Boston, MA, USA), pp. 1-6, 2006.

[11] J. Vaganay, J. Leonard, J. Curcio, and J. Willcox, "Experimental validation of the moving long base line navigation concept," in Autonomous Underwater Vehicles, 2004 IEEE/OES, pp. 59-65, Jun 2004.

[12] A. Bahr, Cooperative Localization for Autonomous Underwater Vehicles. PhD thesis, Massachusetts Institute of Technology, Cambridge, MA, USA, Feb 2009

[13] M. Fallon, G. Papadopoulos, and J. Leonard, "Cooperative AUV navigation using a single surface craft," in Field and Service Robotics (FSR), Jul 2009.

[14] S. E. Webster, L. L. Whitcomb, and R. M. Eustice, "Preliminary results in decentralized estimation for single-beacon acoustic underwater navigation," in Robotics: Science and Systems (RSS), (Zaragoza, Spain), June 2010.

[15] S. Julier and J. Uhlmann, "A counter example to the theory of simultaneous localization and map building," in Robotics and Automation, 2001. Proceedings 2001 ICRA. IEEE International Conference on, vol. 4, pp. 4238 - 4243 vol.4, 2001.

[16] M. F. Fallon, G. Papadopoulos, J. J. Leonard, and N. M. Patrikalakis, "Cooperative AUV navigation using a single maneuvering surface craft," Intl. J. of Robotics Research, vol. 29, pp. 1461-1474, October 2010.

[17] I. Tena Ruiz, S. de Raucourt, Y. Petillot, and D. Lane, "Concurrent mapping and localization using sidescan sonar," Journal of Oceanic Engineering, vol. 29, pp. 442 - 456, Apr. 2004.

[18] I. Tena Ruiz, S. Reed, Y. Petillot, J. Bell, and D. Lane, "Concurren mapping and localization using sidescan sonar for autonomous navigation," in Proc. Int. Symp. on Unmanned Untethered Submersible Technology, 2003.

[19] P. Newman and J. Leonard, "Pure range-only sub-sea SLAM," in IEEE Intl. Conf. on Robotics and Automation (ICRA), vol. 2, pp. 1921 - 1926 vol.2, 14-19 2003.

[20] E. Olson, J. J. Leonard, and S. Teller, "Robust range-only beacon localization," in AUV 2004, June 2004.

[21] A. Kim and R. Eustice, "Pose-graph visual SLAM with geometric model selection for autonomous underwater ship hull inspection," in IEEE/RSJ Intl. Conf. on Intelligent Robots and Systems (IROS), Oct 2009.

[22] I. Mahon, S. Williams, O. Pizarro, and M. Johnson-Roberson, "Efficient view-based SLAM using visual loop closures," IEEE Trans. Robotics, vol. 24, pp. 1002-1014, Oct 2008.

[23] M. Kaess, A. Ranganathan, and F. Dellaert, "iSAM: Incremental smoothing and mapping," IEEE Trans. Robotics, vol. 24, pp. 1365 1378, Dec 2008.

[24] H. Johannsson, M. Kaess, B. Englot, F. Hover, and J. Leonard, "Imaging sonar-aided navigation for autonomous underwater harbor surveillance," in IEEE/RSJ Intl. Conf. on Intelligent Robots and Systems (IROS), (Taipei, Taiwan), Oct 2010

[25] L. Whitcomb, D. Yoerger, H. Singh, and D. Mindell, "Towards precision robotic maneuvering, survey, and manipulation in unstructured undersea environments," in Proc. of the Intl. Symp. of Robotics Research (ISRR), vol. 8, pp. 45-54, 1998.

[26] D. Yoerger, M. Jakuba, A. Bradley, and B. Bingham, "Techniques for deep sea near bottom survey using an autonomous underwater vehicle,' Intl. J. of Robotics Research, pp. 416-429, Jan. 2007.

[27] D. B. Heckman and R. C. Abbott, "An acoustic navigation technique," in IEEE Oceans '73, pp. 591-595, 1973.

[28] M. Hunt, W. Marquet, D. Moller, K. Peal, W. Smith, and R. Spindel, "An acoustic navigation system," Tech. Rep. WHOI-74-6, Woods Hole Oceanographic Institution, 1974.

[29] L. Freitag, M. Grund, S. Singh, J. Partan, P. Koski, and K. Ball, "The WHOI micro-modem: An acoustic communications and navigation system for multiple platforms," in Proceedings of the IEEE/MTS OCEANS Conference and Exhibition, vol. 1, pp. 1086-1092, Sep 2005.

[30] R. M. Eustice, L. L. Whitcomb, H. Singh, and M. Grund, "Experimental results in synchronous-clock one-way-travel-time acoustic navigation for autonomous underwater vehicles," in IEEE Intl. Conf. on Robotics and Automation (ICRA), (Rome, Italy), pp. 4257-4264, Apr 2007.

[31] S. E. Webster, Decentralized single-beacon acoustic navigation: combined communication and navigation for underwater vehicles. $\mathrm{PhD}$ thesis, Johns Hopkins University, Baltimore, MD, USA, June 2010.

[32] H. E. Edgerton, Sonar Images. Englewood Cliffs, NJ: Prentice-Hall, 1986.

[33] M. Cummins and P. Newman, "Probabilistic appearance based navigation and loop closing," in IEEE Intl. Conf. on Robotics and Automation (ICRA), pp. 2042-2048, Apr 2007.

[34] B. Kim, M. Kaess, L. Fletcher, J. Leonard, A. Bachrach, N. Roy, and S. Teller, "Multiple relative pose graphs for robust cooperative mapping," in IEEE Intl. Conf. on Robotics and Automation (ICRA), (Anchorage, Alaska), pp. 3185-3192, May 2010.

[35] A. Howard, "Multi-robot simultaneous localization and mapping using particle filters," in Robotics: Science and Systems (RSS), Jun 2005.

[36] M. F. Fallon, G. Papadopoulos, and J. J. Leonard, "A measurement distribution framework for cooperative navigation using multiple AUVs," in IEEE Intl. Conf. on Robotics and Automation (ICRA), pp. 48034808, May 2010.

[37] M. Benjamin, J. Leonard, H. Schmidt, and P. Newman, "An overview of MOOS-IvP and a brief users guide to the IvP helm autonomy software," Tech. Rep. CSAIL-2009-028, Jun 2009.

[38] T. Schneider and H. Schmidt, "Unified command and control for heterogeneous marine sensing networks," J. of Field Robotics, 2010. 